\title{
Hypoxia-dependent regulation of inflammatory pathways in immune cells
}

\author{
Cormac T. Taylor, ${ }^{1,2,3}$ Glen Doherty, ${ }^{1,4}$ Padraic G. Fallon, ${ }^{5}$ and Eoin P. Cummins ${ }^{1}$
}

'School of Medicine and Medical Science and the Conway Institute, University College Dublin, Belfield, Dublin, Ireland. RCAN, Centre A. Lacassagne, University of Nice-Sophia Antipolis, Nice, France

${ }^{3}$ Centre Scientifique de Monaco (CSM), Monaco. ${ }^{4}$ Centre for Colorectal Disease, St. Vincent's University Hospital, Elm Park, Dublin, Ireland. ${ }^{5}$ Trinity Biomedical Science Institute, Trinity College Dublin, Dublin, Ireland.

\begin{abstract}
Uncontrolled inflammation underpins a diverse range of diseases where effective therapy remains an unmet clinical need. Hypoxia is a prominent feature of the inflammatory microenvironment that regulates key transcription factors including HIF and NF-кB in both innate and adaptive immune cells. In turn, altered activity of the pathways controlled by these factors can affect the course of inflammation through the regulation of immune cell development and function. In this review, we will discuss these pathways and the oxygen sensors that confer hypoxic sensitivity in immune cells. Furthermore, we will describe how hypoxia-dependent pathways contribute to immunity and discuss their potential as therapeutic targets in inflammatory and infectious disease.
\end{abstract}

\section{The relationship between hypoxia and} inflammation

The appearance and accumulation of gaseous molecular oxygen $\left(\mathrm{O}_{2}\right)$ in the Earth's atmosphere approximately 2.3 billion years ago, which occurred as a result of the expansion of oceanic photosynthetic cyanobacteria, resulted in near eradication of the planet's biomass due to its highly reactive chemistry (1). However, a subset of unicellular organisms, which evolved following the incorporation of mitochondria during an early symbiotic event, developed the ability to withstand the toxic effects of oxygen (2). In fact, these primitive organisms adapted to an oxygen-rich environment by developing the capacity to utilize oxygen's inherent chemical energy to increase their metabolic potential. This played a major role in providing the capacity for satisfying the bioenergetic demands required for the subsequent evolution of eukaryotic metazoan life on Earth $(2,3)$. Of note, in early unicellular organisms, the processes of metabolism and primitive immunity shared many common features such as phagocytosis and proteolysis, and therefore there exists an ancient and intimate relationship between these two fundamental processes (4).

$\mathrm{O}_{2}$ plays a vital role in eukaryotic metabolism as the terminal electron acceptor of the mitochondrial electron transport chain, which is responsible for generating the majority of ATP produced by a cell (5). Indeed, eukaryotic cells have developed a strong degree of dependence upon the availability of sufficient levels of $\mathrm{O}_{2}$ to maintain biological activity and remain viable.

Hypoxia occurs when oxygen demand exceeds supply, and as such represents a significant threat to bioenergetic homeostasis and cell survival. A number of recent studies have demonstrated that the microenvironment at sites of inflammation often becomes profoundly hypoxic. Inflammatory pathologies

Conflict of interest: The authors have declared that no conflict of interest exists. Reference information: / Clin Invest. 2016;126(10):3716-3724. doi:10.1172/JCI84433. in which tissue hypoxia has been documented include atherosclerosis, rheumatoid arthritis, obesity, infection, ischemic disease, cancer, and inflammatory bowel disease (6). The occurrence of hypoxia at sites of inflammation is due to a combination of increased oxygen demand and decreased supply. Oxygen consumption is elevated at inflammatory sites due to the high metabolic demand associated with active inflammation, which is necessary to sustain the synthesis of inflammatory mediators, enzymes, and cytokines $(6,7)$. In addition, infiltrating neutrophils at sites of inflammation contribute to hypoxia, as these cells require high levels of oxygen to support the oxidative burst that is used as an endogenous antimicrobial strategy $(8,9)$. Reduced oxygenation of inflamed tissues is of particular relevance in chronic inflammatory diseases of the intestine, such as inflammatory bowel disease, where the development of thrombosis, fibrosis, and associated vascular dysfunction often leads to diminished tissue perfusion $(10,11)$. Therefore, hypoxia is a common feature of the microenvironment of chronically inflamed tissues.

It has recently become clear that, rather than simply being a consequence of inflammation, hypoxia can actively affect inflammatory processes through the regulation of oxygen-sensitive signaling pathways in multiple immune cell subtypes that are either resident within the inflamed tissue or have migrated from the oxygen-rich bloodstream to the hypoxic inflammatory milieu $(6,12)$. In this review, we will discuss these pathways and the implications of their activation for the regulation of immunity and inflammation.

\section{Hypoxia-responsive pathways in inflammation}

Cellular responses to hypoxia are governed by a marked change in the cellular transcriptome (13). Pan-transcriptomic analyses have identified hundreds of hypoxia-responsive genes, with equivalent numbers of genes having increased or decreased expression levels $(14,15)$. Over 15 distinct transcription factors display sensitivity to 


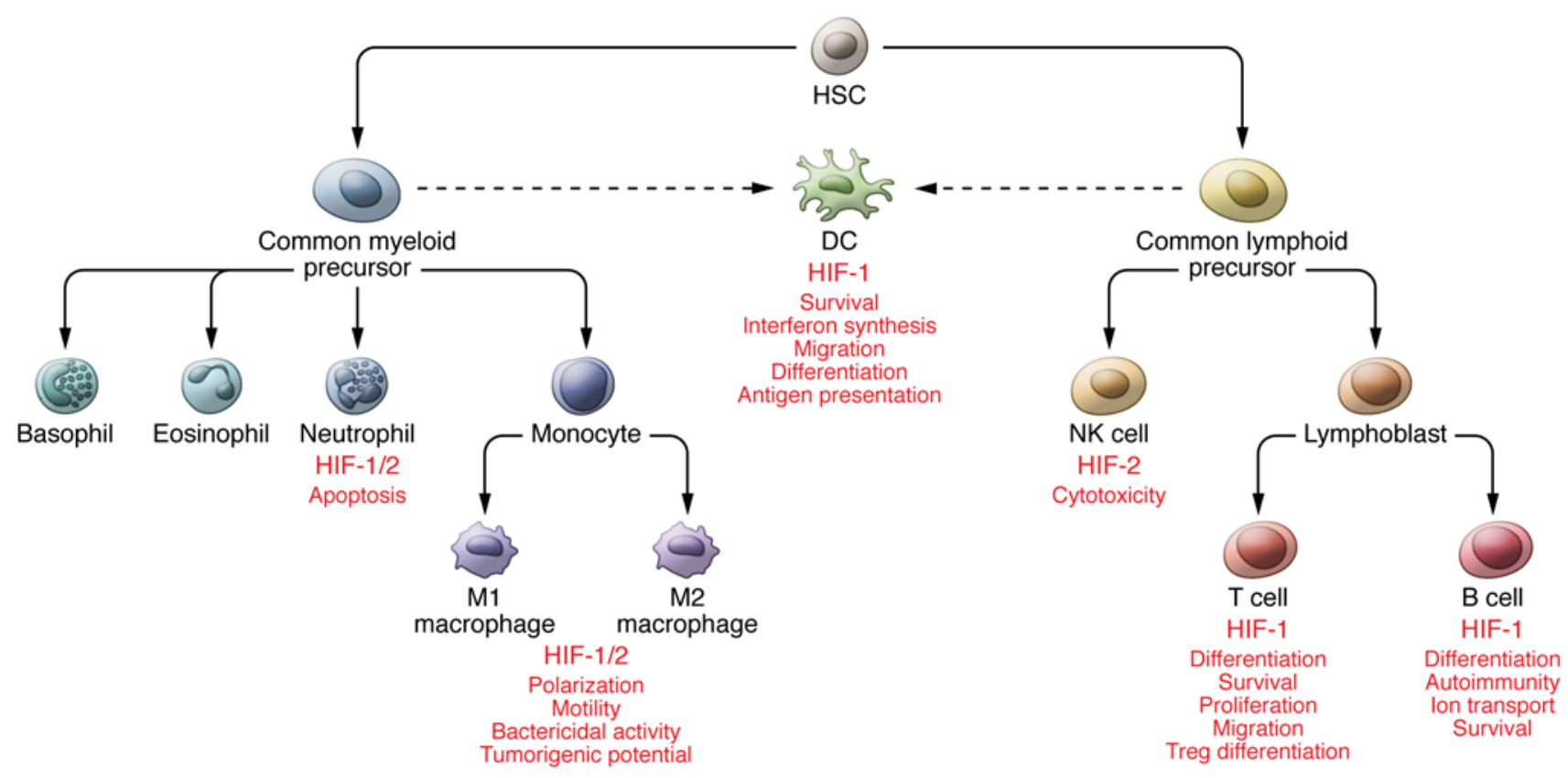

Figure 1. Regulation of immune cells by HIFs. Functional immune cells differentiate from hematopoietic stem cells (HSC). Immune cells are frequently exposed to hypoxia when they enter the hypoxic niche of the inflammatory lesion where HIF can influence differentiation and function (shown in red).

hypoxia and likely contribute, to varying degrees, to driving the global transcriptional response (16). While our understanding of the factors controlling gene repression in hypoxia is currently limited, both HIF and NF-kB are prominent, well-described hypoxiaresponsive transcriptional activators.

Hypoxia-inducible factor. HIF is considered to be a master regulator of the cellular response to hypoxia $(13,17)$. HIF is a heterodimeric transcription factor consisting of a labile $\alpha$ subunit and a stably expressed $\beta$ subunit (HIF-1 $\beta$ /ARNT). Oxygen sensitivity of the HIF $\alpha$ subunit is conferred by a family of dioxygenases termed HIF hydroxylases, which comprises three prolyl hydroxylases (PHD13) and a single asparagine hydroxylase termed the factor inhibiting HIF (FIH) (13, 17-19). In normoxia, when cellular oxygen supply exceeds demand, PHDs hydroxylate HIF on proline residues (Pro-402 and Pro-564 on HIF-1 $\alpha$ ) and target it for ubiquitination, which is mediated by the von Hippel-Lindau (VHL) E3 ubiquitin ligase, and subsequent proteasomal degradation, thereby maintaining HIF in a largely repressed state in normal physiological conditions. A second level of HIF repression is provided by FIH, which hydroxylates HIF on an asparagine residue (Asn-803 in HIF-1 $\alpha$ ), thereby preventing its interaction with the transcriptional co-activating proteins CBP and p300 $(18,19)$. In hypoxia, where oxygen is a limiting factor, the activity of the HIF hydroxylases is reduced, leading to the stabilization of HIF $\alpha$ subunits, which bind to HIF-1 $\beta$ and form stable HIF dimers that are then free to bind to $\mathrm{CBP}$ and $\mathrm{p} 300$, initiating the formation of a functionally active transcriptional complex (18). There are two main transcriptionally active isoforms of HIF, termed HIF-1 and HIF-2, which are differentiated by whether they comprise a HIF- $1 \alpha$ or a HIF-2 $\alpha$ subunit in combination with HIF-1 $\beta$, respectively. HIF-1 and HIF-2 have discrete tissue expression profiles and overlapping but distinct sets of target genes (15). Physiological processes that are under the con- trol of the HIF pathway include metabolism, erythropoiesis, and angiogenesis and, because of its activation in the hypoxic regions of growing tumors, the HIF pathway has been heavily implicated in tumor growth and the progression of cancer (13).

It has recently come to be appreciated that the metabolic status of an immune cell is intimately linked with its phenotype and function (20-22). Therefore, in the context of immunity and inflammation, the sentinel role of HIF in the regulation of metabolic processes is of primary importance. HIF both regulates and is regulated by metabolism. For example, HIF is a strong driver of glycolytic gene expression and a repressor of oxidative phosphorylation (23), while metabolic intermediates including fumarate and succinate can modulate HIF-dependent signaling. Dysregulation of metabolic enzymes involved in the production of these intermediates in the tumor setting are associated with altered HIF-dependent signaling $(24,25)$. In the context of infection, LPS-dependent activation of macrophages leads to metabolic reprogramming, succinate release, and elevated IL- $1 \beta$ production downstream of HIF (26). Therefore, HIF sits in a prime position to link metabolic and immune/inflammatory processes.

\section{HIF and immunity}

Recently, a major role for HIF has emerged in multiple aspects of immune cell function and survival (Figure 1). This is perhaps unsurprising considering that many immune cells leave the oxygen-rich vasculature as they migrate to hypoxic sites of inflammation, where they carry out their immunological function and are therefore regularly subject to a sharp drop in oxygen levels and must adapt accordingly.

While HIF-1 appears to be expressed in all immune cell types, HIF-2 is more selectively expressed in cells such as neutrophils, NK cells, tumor-associated macrophages, and activated 


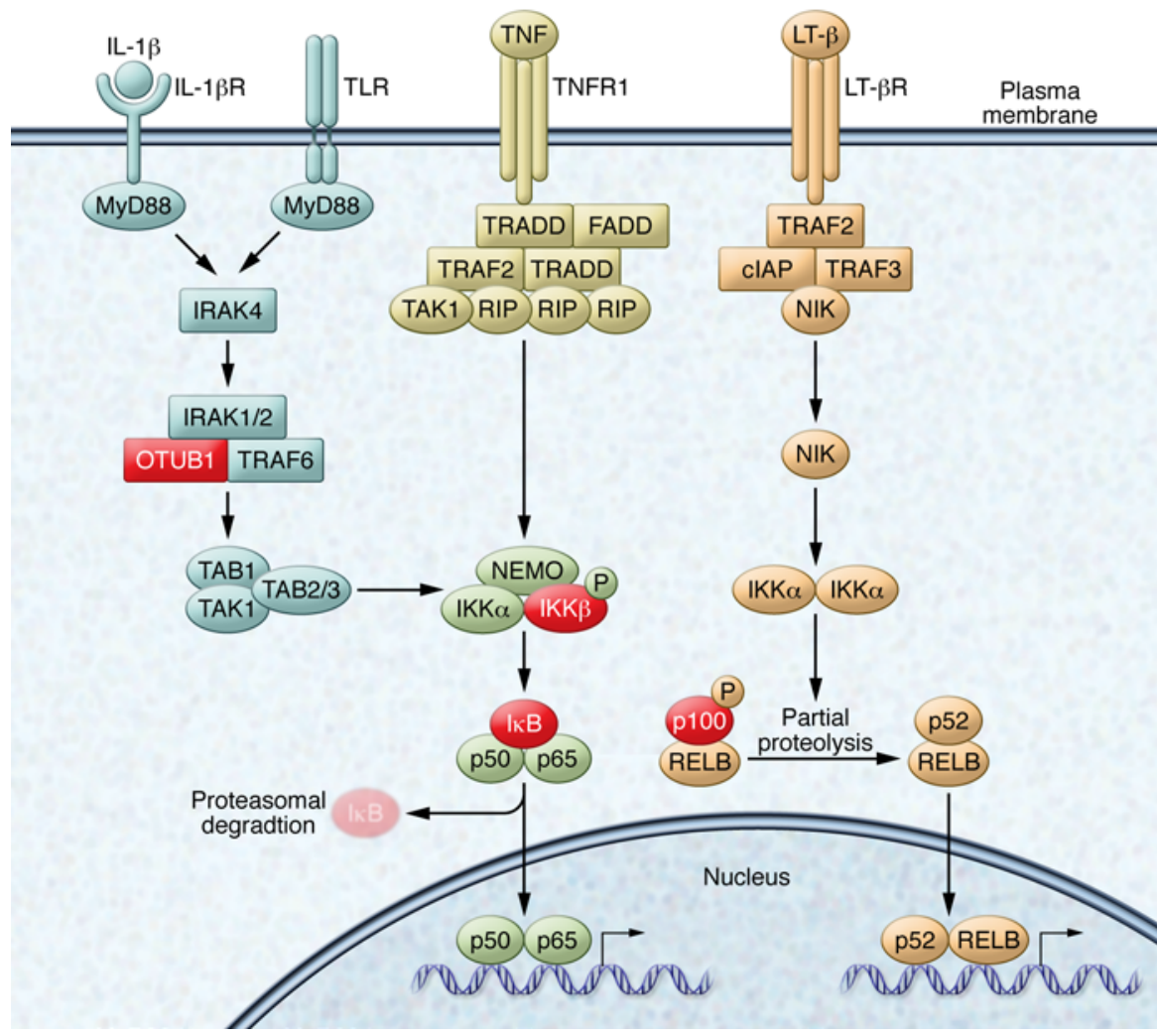

Figure 2. Potential sites for functional hydroxylation in the NF- $\kappa$ B pathway. NF- $\kappa \mathrm{B}$-dependent signaling can be activated by several proinflammatory factors that bind to discrete membrane-associated receptors and activate discrete pathways. Putative sites for functional hydroxylation in these pathways are outlined in red.

T cells under conditions of hypoxia (22). Studies utilizing transgenic mice, in which the genes encoding individual HIF subunits have been deleted in distinct immune cell subtypes, have elucidated some of the specific roles of HIF-1 $\alpha$ and HIF-2 $\alpha$ in immunity and inflammation. A dominant and consistent theme that has evolved is that HIFs play a central role in the regulation of immune cell development and function; consequently, HIFs should be considered key regulators of immunity and inflammation. These studies revealed cell type-specific roles for HIF in the regulation of a number of processes central to immune cell activity (27). While our understanding of the role of HIFs in immune cell function is expanding, it is notable that during inflammation, hypoxia occurs not in isolation but in the complex milieu of the inflammatory lesion. As such, a number of other factors such as the presence of cytokines and chemokines will combine with hypoxia to potently regulate immune cell development and function and the ultimate effects of hypoxia will depend upon which of these co-stimuli are present.

\section{HIF in innate immunity}

Macrophages can differentiate into M1 or M2 phenotypes, which are associated with first-line (proinflammatory) antibacterial defense and wound healing (antiinflammatory) respectively. Additionally, these macrophage phenotypes employ distinct metabolic strategies. M1 macrophages are largely reliant on glycolysis, while M2 macrophages are largely reliant on oxidative phosphorylation for ATP production (28). Not surprisingly, both HIF-1 and HIF2 play important regulatory functional roles in the control of motility, bactericidal activity, and tumorigenic potential in macrophages (29-31). However, it remains unclear exactly how HIF-1 and HIF-2 participate in the skewing of macrophages towards an M1 or M2 phenotype (20, 22). While HIF-1-dependent glycolysis favors polarization to an M1 phenotype, recent work has indicated that M2 polarization is independent of either HIF isoform, at least in a model of sterile tissue damage (32). Therefore, while it is likely that HIF activation contributes to macrophage polarization, the specific roles of individual HIF isoforms require further investigation.

In neutrophils, HIF-1 and HIF-2 are critical in the control of cell survival and apoptosis. HIF-1 promotes neutrophil survival and bactericidal activity $(33,34)$. Similarly, HIF-2 appears to support neutrophilic inflammation through the promotion of cell survival (35). Of interest, HIF-2 expression in neutrophils is elevated in patients with inflammatory disease (35). Therefore, in the context of inflammation, HIF-1/2 activation in neutrophils can be considered to be largely proinflammatory.

In dendritic cells (DCs), HIF-1 has been shown to promote cell survival, interferon synthesis, differentiation, and migration (36-38). Furthermore, recent studies have suggested a role for HIF-1 $\alpha$ in determining the antigen-presenting function of DCs. Additionally, HIF-1 has been implicated in promoting Treg expansion while limiting the expansion of $\mathrm{CD}^{+} \mathrm{T}$ cells $(39,40)$. These latter studies support a predominantly antiinflammatory function for HIF-1 in DCs. Little is known about the expression or function of HIF-2 in DCs. In NK cells, HIF-2 limits cellular cytotoxicity, indicating an antiinflammatory role (41). Therefore, HIFs play a key role in multiple aspects of innate immune cell development and function.

\section{HIF and barrier function}

Epithelial cells are important and often-neglected innate immune cells that play a key role as a first line of defense against external microbes in tissues such as the lung, skin, and gastrointestinal tract. Indeed, epithelial barrier function is a key aspect of innate immunity. In epithelial cells of both the intestine and the skin, HIF-1 and HIF-2 have been shown to be crucial to regulating barrier function and wound healing capacity, although the different isoforms may perform opposing roles (42-45). Strong data now exist demonstrating that HIF-1 is associated with improved epithelial barrier function in the gastrointestinal tract, making HIF a potential target in inflammatory bowel disease, which is associated with epithelial barrier dysfunction (46). Therefore HIFs are key regulators of epithelial barrier function, an important aspect of innate immunity. 

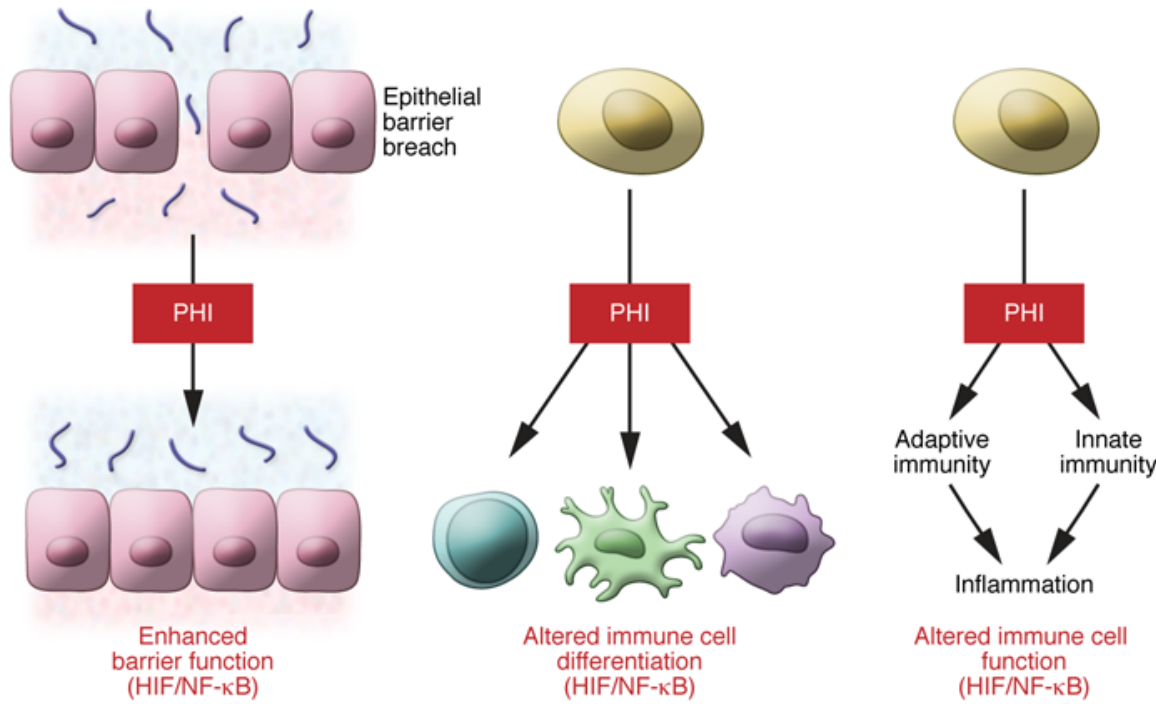

Figure 3. Mechanisms of therapeutic action of hydroxylase inhibitors in inflammation. Pharmacological hydroxylase inhibition influences inflammatory processes by (1) enhancing barrier function (in infectious inflammation), (2) altering immune cell differentiation, and (3) altering innate and adaptive immune cell function.

\section{HIF in adaptive immunity}

The important role that HIF plays in the regulation of the adaptive immune response and the implications of this regulation for inflammation and cancer have been recently reviewed (21, 22). Depending on environmental cues, $\mathrm{CD} 4^{+} \mathrm{T}$ cells can differentiate into Tregs, Th1, Th2, or Th17 cells, which have distinct immunological functions. HIF-1 has been reported to regulate T cell survival, proliferation, and differentiation (47). HIF-1 activation promotes a metabolic shift to glycolysis as well as altered transcriptional responses in differentiating $\mathrm{T}$ cells (via regulation of the nuclear hormone receptor ROR $\gamma \mathrm{t}$ and the transcriptional regulator FOXP3), creating an environment favorable for the differentiation to Th17 cells rather than Tregs $(21,48)$. In activated T cells, HIF-1 promotes cytolytic, migratory, and co-stimulatory properties $(22,49)$, although HIF-1 has recently been reported to negatively regulate Th1 cell function in some conditions (50). The impact of HIF-1 in Treg function is less clear. One study reports that HIF-1-driven FOXP3 is required for Treg function and cells lacking HIF-1 $\alpha$ have reduced antiinflammatory capacity and lose their ability to control inflammation (51). A separate study demonstrated that VHL depletion in Tregs leads to HIF-1 activation, which drives their transformation to Th1 effector killer cells, thereby promoting inflammation (52). In summary, HIF-1 plays a key role in $\mathrm{T}$ cell differentiation by promoting glycolytic metabolism and directing key aspects of $\mathrm{T}$ cell function, and as such is a key mediator of adaptive $\mathrm{T}$ cell-mediated immunity.

In terms of B cell biology, HIF-1 $\alpha^{-/-}$Rag2 ${ }^{-/}$chimeric mice display B cell lineage defects, including abnormal B1-like lymphocytes and evidence of autoimmunity (53). More recently, HIF- $1 \alpha$ has been shown to be important for the expression of TASK-2 potassium channels in B cells. These channels are vital for a number of cellular functions including proliferation, survival, and cytokine production (54). Furthermore, HIF-1 $\alpha$ is critical for crosstalk between mature B cells (plasma cells) and the microenvironment in tumors (55). Finally, depletion of HIF-1 $\beta$ leads to alterations in homeostasis and hematopoietic stem cell viability (56). Therefore, in the context of adaptive immune cell activity, HIFs play a key role, which appears to be predominantly antiinflammatory for $\mathrm{T}$ cells and prosurvival for B cells.

The studies discussed above provide strong evidence that HIF-1 plays a complex and pleiotropic role in cells of the immune system. A consistent observation throughout these studies is that the effect of genetic HIF depletion from both innate and adaptive immune cell types markedly alters multiple aspects of their function. The discrete and common roles of HIF$1 \alpha$ and HIF- $2 \alpha$ in the differentiation and function of certain immune cell subtypes are summarized in Figure 1. The conclusion from the studies outlined above is that the HIF pathway should be considered an important regulator of both innate and adaptive immune cell function and the inflammatory processes that are activated during inflammation.

$N F-\kappa B$. NF- $\mathrm{KB}$ is a hypoxia-sensitive transcription factor that plays an important role in the control of the inflammatory response (57). The NF-кB signaling pathway consists of distinct canonical and noncanonical branches that play a complex and pleiotropic role in the control of inflammation and can regulate both pro- and antiinflammatory processes, depending upon the context. The canonical (but not the noncanonical) branch of the NF-кB pathway has been demonstrated to respond to hypoxia (58). NF- $\mathrm{KB}$ serves as a master regulator of the immune response through the regulation of key aspects of immune cell function in macrophages, epithelial cells, neutrophils, DCs, T cells, and B cells. The potent role of NF-KB in the regulation of immunity and inflammation has been extensively reviewed elsewhere and will not be covered in detail here (59). For the purpose of this review, we will focus on our evolving understanding of the mechanistic link between hypoxia and NF- $\mathrm{\kappa B}$ activity.

As will be discussed in more detail below, the same hydroxylases responsible for conferring oxygen dependence upon the HIF pathway also regulate hypoxia-dependent NF- $\mathrm{kB}$ activity (60), although the exact mechanisms by which this occurs remain to be fully elucidated. Of interest, a number of hydroxylation sites have been identified on components of the NF- $\mathrm{kB}$ pathway, supporting a possible role for functional, oxygen-dependent hydroxylation outside of the HIF pathway (Figure 2; refs. 60-64). While hypoxia or pharmacological hydroxylase inhibition causes a moderate increase in basal NF- $\kappa \mathrm{B}$ activity in several cell types, it suppresses the activation of NF- $\mathrm{kB}$ activity in cells following treatment with cytokines such as IL-1 $\beta$ or bacterial products such as LPS. These effects reflect a complex regulatory role for hypoxia in relation to the NF- $\mathrm{kB}$ pathway $(65,66)$. However, it is now clear that along with HIF, NF- $\mathrm{KB}$ is a second hypoxia-responsive transcription 
factor that plays a key role in the regulation of inflammation and immunity through its prominent role in the control of immune cell function. Whether NF- $\mathrm{BB}$ induces a pro- or antiinflammatory response depends on the cell type and the degree and duration of its activity in the context of a specific inflammatory lesion.

While both HIF and NF- $\mathrm{KB}$ play distinct roles in terms of regulating inflammatory processes, the two pathways have also been shown to display a significant degree of interdependence (67-70). For example, HIF transcription can be upregulated by NF- $\kappa \mathrm{B}$ through its binding to a response element present in the HIF1A promoter (71-74); thus, NF- $\kappa \mathrm{B}$ activation may serve to amplify the HIF response under conditions of hypoxic inflammation. Conversely, in neutrophils, HIF has been demonstrated to regulate $\mathrm{NF}-\kappa \mathrm{B}$ activity, although this appears to be a more cell type-specific role, as HIF-dependent NF- $\kappa \mathrm{B}$ activity has not been reported in other cell types (33).

In summary, HIF and NF- $\mathrm{BB}$ are two interdependent hypoxia-responsive transcription factors that play a vital role in the control of immune cell function and perform an important bridging role between the microenvironment, metabolism, and the control of immune cell function. Importantly, a common feature of HIF and $\mathrm{NF}-\kappa \mathrm{B}$ is their regulation by oxygen-sensing hydroxylases, thus identifying these oxygen sensors as potentially important therapeutic targets in inflammatory disease.

\section{Regulation of immune cell function by PHDs}

The identification of hydroxylases as the sensors underpinning oxygen dependence of the HIF pathway was first reported in $2001(75,76)$. These studies demonstrated that oxygen-dependent proline hydroxylation of HIF by a family of hydroxylases led to its ubiquitination by the pVHL ubiquitin ligase and subsequent proteasomal degradation in normoxia (18). Another study identified FIH as a second, oxygen-dependent hydroxylase that targets HIF $(77,78)$. Subsequent studies demonstrated that while HIF is clearly the major pathway regulated by hydroxylation, signaling components of other pathways such as NF- $\mathrm{BB}$ are also subject to enzymatic hydroxylation, although the functional consequences of hydroxylation for non-HIF targets remains an area under investigation $(62-64,79)$.

Because of the role of hydroxylases in the regulation of hypoxia-responsive transcription factors, these enzymes also play a key role in the regulation of immune cell activity (27). While our understanding of the role of HIF hydroxylases in the regulation of immune cell function is far from complete, recent studies investigating individual HIF hydroxylase isoforms in discreet immune and epithelial cell subtypes have identified isoform-specific roles (80-89). Notably, these phenotypes are not fully accounted for by downstream HIF-dependent effects, further supporting the existence of alternative hydroxylase-regulated pathways such as $\mathrm{NF}-\kappa \mathrm{B}$ in immune cells.

In macrophages, PHD2 loss or haplodeficiency alters M1/ M2 cell differentiation in an NF-kB-dependent manner $(80,81)$, whereas PHD3 loss alters apoptosis and proinflammatory activity $(82,83)$. In neutrophils, PHD3 regulates apoptosis, although this effect appears to be NF- $\mathrm{BB}$ independent (84). In intestinal epithelial cells, PHD1 and PHD3 have been linked with the regulation of apoptosis and tight-junction integrity, respectively ( 85 ,
86). Interestingly, the expression of these enzymes was reported to be altered in inflammatory bowel disease, where the intestinal barrier is significantly perturbed (85-87). In keratinocytes, loss of PHD2 alters cell migration and subsequent wound healing in a HIF-dependent manner (88). In lymphocytes, loss of PHD2 is associated with altered cytokine production and immune activity (89). Therefore, multiple studies support a key role for hydroxylases and their downstream targets in the regulation of immunity and inflammation.

\section{Hydroxylase-independent pathways activated in hypoxia}

It is worth noting that in addition to HIF and NF- $\mathrm{BB}$, a number of other important hypoxia-sensitive regulators of inflammation are also activated at sites of inflammation, independent of altered hydroxylase activity, and are likely contributors to control of the overall inflammatory state. These regulators do not directly sense changes in oxygen but are sensitive to the sequelae downstream of hypoxia-dependent changes in glucose transport, ATP availability, acidosis, oxidative stress, or altered levels of metabolic intermediates. Some examples are given below.

AMPK is a metabolic sensor that is activated in response to ATP depletion that occurs as a result of the metabolic crisis caused by hypoxia, and has recently emerged as a key regulator of inflammatory cell activity (90). The glucocorticoid receptor (GR), which plays a key role in the activation of antiinflammatory genes and the suppression of proinflammatory genes, is activated in response to hypoxia and likely contributes to the overall control of inflammation, although the mechanisms underpinning the activation of the GR by hypoxia remain unclear $(91,92)$. Members of the cAMP response element-binding protein (CREB) family, including CREB and ATF4, have been shown to be regulated by hypoxia and can in turn regulate the expression of inflammatory genes in immune cells $(93,94)$. The mTOR pathway, which is activated in response to hypoxia, likely plays a key role in immune cell activity during inflammation (95). Acidosis, which is a feature of the inflammatory microenvironment, is a known regulator of the hypoxic response (96) Indeed, lactate, which is produced during anaerobic respiration, has recently been reported to induce a hypoxic response independent of HIF, via NMYC downstreamregulated gene 2 (NDRG2) and Raf/ERK signaling (97).

Therefore, multiple hypoxia-sensitive but hydroxylase-independent pathways in addition to HIF and NF-אB likely contribute to the complex and multifaceted effects of hypoxia on immune cell activity during active inflammation. The complexity of these interactions make the crosstalk between hypoxia and inflammatory pathways an attractive topic for developing a systems biology approach to advance our understanding of the temporal and dynamic aspects of this relationship (98).

\section{Intermittent hypoxia and inflammation}

Most studies into hypoxia-dependent transcription have utilized models of continuous or sustained hypoxia, whereas in vivo it is likely that cells experience bouts of fluctuating hypoxia and reoxygenation, as oxygen consumption and delivery is a highly dynamic process both in health and disease. In a number of conditions (exemplified by obstructive sleep apnea syndrome, of which sys- 
temic inflammation is a feature), cells clearly experience a more intermittent profile of hypoxia and reoxygenation (99). Indeed, it is likely that other hypoxic pathologies including chronic inflammation and cancer also involve exposure to an intermittent rather than a sustained pattern of hypoxia. Intermittent hypoxia represents a significantly different physiological stimulus than sustained hypoxia, as it also involves repeated exposure to bouts of reoxygenation and associated oxidative stress. Studies into the impact of intermittent hypoxia on cellular signaling pathways have implicated both HIF and NF- $\kappa$ B pathways in response to intermittent hypoxia, although sustained hypoxia favors activation of the HIF pathway while intermittent hypoxia is more selective for the activation of $\mathrm{NF}-\kappa \mathrm{B}$-driven inflammation, likely as a result of oxidative stress (99-102). Little is known about the differential effects of intermittent and sustained hypoxia on immune cell function, although this is likely of importance for a full understanding of the impact of physiologically relevant patterns of hypoxia on inflammation and immunity.

\section{Preclinical studies of hydroxylase inhibitors}

The evidence outlined above, which supports key roles for hypoxia- and hydroxylase-sensitive transcriptional pathways in immunity and inflammation, indicates that pharmacological interference with these pathways would likely have immunological consequences. However, given the complex and pleiotropic roles of hypoxia-responsive pathways in distinct immune cell types, a prediction of whether hypoxia and/or hydroxylase inhibition would ultimately be pro- or antiinflammatory is difficult to make without testing the impact of pharmacological hydroxylase inhibition in vivo.

Studies implicating hypoxia-responsive pathways in inflammatory disease are supported by data demonstrating the impact of targeting the HIF pathway with pharmacological hydroxylase inhibitors for the treatment of inflammatory disease $(12,103)$. Initial work in this area demonstrated a beneficial effect of hydroxylase inhibition on disease progression in mouse models of colitis (104, 105). This protective effect was found to be due primarily to the promotion of intestinal epithelial barrier function through the inhibition of apoptosis and the expression of genes that enhance barrier function $(46,106)$. Subsequent studies demonstrated a broader antiinflammatory effect of pharmacological hydroxylase inhibition in diseases such as ischemic acute kidney injury and sepsis, leading to the conclusion that other mechanisms of therapeutic action may also be involved $(65,107)$. Indeed, recent studies have implicated hydroxylase inhibitors in the suppression of cytokine-activated $\mathrm{NF}-\kappa \mathrm{B}-$ dependent proinflammatory pathways, which may underpin a more general antiinflammatory effect of these drugs (64). Therefore, while hypoxia-sensitive pathways clearly play a complex and multifaceted role in the regulation of immunity and inflammation, the pharmacological activation of these pathways through the application of hydroxylase inhibitors provides a strong, net antiinflammatory effect in multiple models (Figure 3). Future clinical studies are likely to be directed towards investigating the potential of hydroxylase inhibitors as a new class of antiinflammatory agents.

In addition to reported antiinflammatory effects, pharmacological hydroxylase inhibition has been shown to ameliorate symptoms associated with infectious disease (108). For example, mice treated with the PHD inhibitor dimethyloxalylglycine, $\mathrm{N}$-(me- thoxyoxoacetyl)-glycine methyl ester (DMOG) are protected in a murine model of respiratory infection with the gram-negative opportunistic pathogen Pseudomonas aeruginosa (109). Consistent with these findings, loss of HIF-1 $\alpha$ in mice increases susceptibility to $P$. aeruginosa-mediated keratitis (110). In other models of infection including Clostridium, Streptococcus, Staphylococcus, and Acinetobacter species, HIF has also been largely found to be protective against pathogens (111-115). Given the evolving issue of antimicrobial resistance in multiple pathogens, future studies will undoubtedly expand on the fascinating potential of targeting host oxygen-sensing pathways for the purpose of regulating host immunity in infectious disease.

\section{Clinical potential of hydroxylase inhibitors}

Hydroxylase inhibitors have recently been entered into clinical trials for the promotion of erythropoiesis in models of chronic kidney disease-associated anemia (116-118). In these studies, the delivery of systemic doses of hydroxylase inhibitors was well tolerated by patients, paving the way for the possible use of these reagents for the treatment of inflammatory disorders and/or infectious disease. Notably, while systemic exposure of a patient to a hydroxylase inhibitor would be beneficial in anemia in order to promote erythropoiesis, the local delivery of these drugs would be desirable in the treatment of tissue-specific inflammation in order to avoid unwanted systemic effects such as erythropoiesis. Such a targeted delivery-based approach has recently been demonstrated in a murine model of colitis, in which localized delivery of the hydroxylase inhibitor DMOG to the colon using an emulsion-based drug delivery system provided tissue protection at lower doses than the doses needed for systemic delivery and without associated systemic erythropoiesis (119). Mechanisms for targeted colonic delivery (e.g., Multi Matrix System [MMX]) of antiinflammatory agents such as aminosalicylates (5ASA) and budesonide are already used in the clinical care of the inflammatory disorder ulcerative colitis (120, 121). These studies provide proof of concept for the treatment of intestinal inflammation based on local release formulations to maximize drug concentrations in diseased tissue while limiting any potential systemic side effects.

\section{Perspectives}

Since the identification of HIF as a ubiquitous regulator of the cellular transcriptional response to hypoxia and the subsequent identification of hydroxylases as the key oxygen sensors in this pathway, our appreciation of the role of hypoxia as a driver of immunity and inflammation has expanded exponentially. The therapeutic payoff for a quarter of a century of basic research in this field is reflected by the recent entry to the clinic of hydroxylase inhibitors for the treatment of anemia. The potential for repurposing these drugs for inflammatory diseases such as inflammatory bowel disease, rheumatoid arthritis, and ischemia is a real possibility in the near future.

Some key aspects of the biology of hypoxia-dependent pathways in inflammation require further investigation. These include extensive characterization of the safety and side effects of systemically administered hydroxylase inhibitors and the possibility of developing more targeted, tissue-specific delivery systems. 
The differential roles of HIF-1- and HIF-2-dependent activity in immune cell subpopulations in both resting and activated states requires further investigation, particularly using comparative studies. Furthermore, the association between hypoxia and other key aspects of the inflammatory microenvironment such as the prevailing cytokine/chemokine milieu and altered carbon dioxide levels and associated changes in $\mathrm{pH}$ need to be considered (122, 123). Finally, comparative studies of intermittent versus sustained patterns of hypoxia in immune cells will strengthen our understanding of the role of physiologically relevant patterns of hypoxic exposure during inflammation. The near future will provide us with these and other exciting new insights into the ancient association between hypoxia, metabolism, and immunity, which will lead to the development of new therapeutic opportunities for inflammatory and infectious disease.

\section{Acknowledgments}

The authors work is supported by grants from Science Foundation Ireland, the European Union, and financial support from the University College Dublin School of Medicine.

Address correspondence to: Cormac T. Taylor, UCD Conway Institute, University College Dublin, Belfield, Dublin 4, Ireland. Phone: 353.1.716.6732; E-mail: cormac.taylor@ucd.ie.
1. Lyons TW, Reinhard CT, Planavsky NJ. The rise of oxygen in Earth's early ocean and atmosphere. Nature. 2014;506(7488):307-315.

2. O'Malley MA. Endosymbiosis and its implications for evolutionary theory. Proc Natl Acad Sci U S A. 2015;112(33):10270-10277.

3. Taylor CT, McElwain JC. Ancient atmospheres and the evolution of oxygen sensing via the hypoxia-inducible factor in metazoans. Physiology (Bethesda). 2010;25(5):272-279.

4. Broderick NA. A common origin for immunity and digestion. Front Immunol. 2015;6:72.

5. Taylor CT. Mitochondria and cellular oxygen sensing in the HIF pathway. Biochem J. 2008;409(1):19-26.

6. Eltzschig HK, Carmeliet P. Hypoxia and inflammation. N Engl J Med. 2011;364(7):656-665.

7. Lalonde C, Demling RH, Goad ME. Tissue inflammation without bacteria produces increased oxygen consumption and distant organ lipid peroxidation. Surgery. 1988;104(1):49-56.

8. Campbell EL, Colgan SP. Neutrophils and inflammatory metabolism in antimicrobial functions of the mucosa. J Leukoc Biol. 2015;98(4):517-522.

9. Campbell EL, et al. Transmigrating neutrophils shape the mucosal microenvironment through localized oxygen depletion to influence resolution of inflammation. Immunity. 2014;40(1):66-77.

10. Wakefield AJ, et al. Pathogenesis of Crohn's disease: multifocal gastrointestinal infarction. Lancet. 1989;2(8671):1057-1062.

11. Flück K, Fandrey J. Oxygen sensing in intestinal mucosal inflammation. Pflugers Arch. 2016;468(1):77-84.

12. Scholz CC, Taylor CT. Targeting the HIF pathway in inflammation and immunity. Curr Opin Pharmacol. 2013;13(4):646-653.

13. Semenza GL. Hypoxia-inducible factors in physiology and medicine. Cell. 2012;148(3):399-408.

14. Leonard MO, Cottell DC, Godson C, Brady HR, Taylor CT. The role of HIF-1 alpha in transcriptional regulation of the proximal tubular epithelial cell response to hypoxia. J Biol Chem. 2003;278(41):40296-40304.

15. Mole DR, et al. Genome-wide association of hypoxia-inducible factor (HIF)-1alpha and HIF-2alpha DNA binding with expression profiling of hypoxia-inducible transcripts. J Biol Chem. 2009;284(25):16767-16775.

16. Cummins EP, Taylor CT. Hypoxia-responsive transcription factors. Pflugers Arch. 2005;450(6):363-371.
17. Semenza GL. Hypoxia-inducible factor 1: master regulator of $\mathrm{O}_{2}$ homeostasis. Curr Opin Genet Dev. 1998;8(5):588-594.

18. Kaelin WG, Ratcliffe PJ. Oxygen sensing by metazoans: the central role of the HIF hydroxylase pathway. Mol Cell. 2008;30(4):393-402.

19. Kaelin WG. Proline hydroxylation and gene expression. Annu Rev Biochem. 2005;74:115-128.

20. Galván-Peña S, O’Neill LA. Metabolic reprograming in macrophage polarization. Front Immunol. 2014;5:420.

21. Barbi J, Pardoll D, Pan F. Metabolic control of the Treg/Th17 axis. Immunol Rev. 2013;252(1):52-77.

22. Palazon A, Goldrath AW, Nizet V, Johnson RS. HIF transcription factors, inflammation, and immunity. Immunity. 2014;41(4):518-528.

23. Semenza GL. HIF-1 mediates metabolic responses to intratumoral hypoxia and oncogenic mutations. JClin Invest. 2013;123(9):3664-3671.

24. Selak MA, et al. Succinate links TCA cycle dysfunction to oncogenesis by inhibiting HIF-alpha prolyl hydroxylase. Cancer Cell. 2005;7(1):77-85.

25. Isaacs JS, et al. HIF overexpression correlates with biallelic loss of fumarate hydratase in renal cancer: novel role of fumarate in regulation of HIF stability. Cancer Cell. 2005;8(2):143-153.

26. Tannahill GM, et al. Succinate is an inflammatory signal that induces IL- $1 \beta$ through HIF-1 $\alpha$. Nature. 2013;496(7444):238-242.

27. Cummins EP, Keogh CE, Crean D, Taylor CT. The role of HIF in immunity and inflammation. Mol Aspects Med. 2016;47-48:24-34.

28. Mills EL, O’Neill LA. Reprogramming mitochondrial metabolism in macrophages as an anti-inflammatory signal. Eur J Immunol. 2016;46(1):13-21.

29. Cramer T, et al. HIF-1alpha is essential for myeloid cell-mediated inflammation. Cell. 2003;112(5):645-657.

30. Talks KL, et al. The expression and distribution of the hypoxia-inducible factors HIF-1alpha and HIF-2alpha in normal human tissues, cancers, and tumor-associated macrophages. Am J Pathol. 2000;157(2):411-421.

31. Imtiyaz HZ, et al. Hypoxia-inducible factor 2alpha regulates macrophage function in mouse models of acute and tumor inflammation. JClin Invest. 2010;120(8):2699-2714.

32. Gondin J, et al. Myeloid HIFs are dispensable for resolution of inflammation during skeletal muscle regeneration. J Immunol. 2015;194(7):3389-3399.

33. Walmsley SR, et al. Hypoxia-induced neutrophil survival is mediated by HIF-1alpha- dependent NF-kappaB activity. J Exp Med. 2005;201(1):105-115.

34. Peyssonnaux C, et al. HIF-1alpha expression regulates the bactericidal capacity of phagocytes. $J$ Clin Invest. 2005;115(7):1806-1815.

35. Thompson AA, et al. Hypoxia-inducible factor $2 \alpha$ regulates key neutrophil functions in humans, mice, and zebrafish. Blood. 2014;123(3):366-376.

36. Naldini A, et al. Hypoxia affects dendritic cell survival: role of the hypoxia-inducible factor-1 $\alpha$ and lipopolysaccharide. J Cell Physiol. 2012;227(2):587-595.

37. Wobben R, Hüsecken Y, Lodewick C, Gibbert K, Fandrey J, Winning S. Role of hypoxia inducible factor- $1 \alpha$ for interferon synthesis in mouse dendritic cells. Biol Chem. 2013;394(4):495-505.

38. Köhler T, Reizis B, Johnson RS, Weighardt H, Förster I. Influence of hypoxia-inducible factor $1 \alpha$ on dendritic cell differentiation and migration. Eur J Immunol. 2012;42(5):1226-1236.

39. Flück K, Breves G, Fandrey J, Winning S. Hypoxia-inducible factor 1 in dendritic cells is crucial for the activation of protective regulatory T cells in murine colitis. Mucosal Immunol. 2016;9(2):379-390.

40. Hammami A, Charpentier T, Smans M, Stäger S. IRF-5-mediated inflammation limits CD ${ }^{+}$ T cell expansion by inducing HIF- $1 \alpha$ and impairing dendritic cell functions during Leishmania infection. PLoS Pathog. 2015;11(6):e1004938.

41. Zhang J, et al. Hypoxia-inducible factor- $2 \alpha$ limits natural killer t cell cytotoxicity in renal ischemia/reperfusion injury. J Am Soc Nephrol. 2016;27(1):92-106.

42. Karhausen J, Furuta GT, Tomaszewski JE, Johnson RS, Colgan SP, Haase VH. Epithelial hypoxia-inducible factor-1 is protective in murine experimental colitis. JClin Invest. 2004;114(8):1098-1106.

43. Rezvani HR, et al. Loss of epidermal hypoxia-inducible factor- $1 \alpha$ accelerates epidermal aging and affects re-epithelialization in human and mouse. J Cell Sci. 2011;124(Pt 24):4172-4183.

44. Cowburn AS, Alexander LE, Southwood M, Nizet V, Chilvers ER, Johnson RS. Epidermal deletion of HIF- $2 \alpha$ stimulates wound closure. J Invest Der matol. 2014;134(3):801-808.

45. Ramakrishnan SK, Shah YM. Role of intestinal HIF-2 $\alpha$ in health and disease. Annu Rev Physiol. 2016;78:301-325.

46. Colgan SP, Taylor CT. Hypoxia: an alarm signal during intestinal inflammation. Nat Rev Gastroenterol Hepatol. 2010;7(5):281-287. 
47. Biju MP, Neumann AK, Bensinger SJ, Johnson RS, Turka LA, Haase VH. Vhlh gene deletion induces Hif-1-mediated cell death in thymocytes. Mol Cell Biol. 2004;24(20):9038-9047.

48. Dang EV, et al. Control of $\mathrm{T}(\mathrm{H}) 17 / \mathrm{T}(\mathrm{reg})$ balance by hypoxia-inducible factor 1 . Cell. 2011;146(5):772-784.

49. Finlay DK, et al. PDK1 regulation of mTOR and hypoxia-inducible factor 1 integrate metabolism and migration of $\mathrm{CD}^{+} \mathrm{T}$ cells. J Exp Med. 2012;209(13):2441-2453.

50. Shehade H, Acolty V, Moser M, Oldenhove G. Cutting edge: hypoxia-inducible factor 1 negatively regulates Th1 function. J Immunol. 2015;195(4):1372-1376.

51. Clambey ET, et al. Hypoxia-inducible factor-1 alpha-dependent induction of FoxP3 drives regulatory T-cell abundance and function during inflammatory hypoxia of the mucosa. Proc Natl Acad Sci U S A. 2012;109(41):E2784-E2793.

52. Lee JH, Elly C, Park Y, Liu YC. E3 ubiquitin ligase VHL regulates hypoxia-inducible factor- $1 \alpha$ to maintain regulatory $\mathrm{T}$ cell stability and suppressive capacity. Immunity. 2015;42(6):1062-1074.

53. Kojima H, et al. Abnormal B lymphocyte development and autoimmunity in hypoxia-inducible factor 1alpha -deficient chimeric mice. Proc Natl Acad Sci USA. 2002;99(4):2170-2174.

54. Shin DH, et al. HIF-1 $\alpha$-mediated upregulation of TASK- $2 \mathrm{~K}^{+}$channels augments $\mathrm{Ca}^{2+}$ signaling in mouse B cells under hypoxia. J Immunol. 2014;193(10):4924-4933.

55 . Borsi E, et al. HIF-1 $\alpha$ inhibition blocks the cross talk between multiple myeloma plasma cells and tumor microenvironment. Exp Cell Res. 2014;328(2):444-455.

56 . Krock BL, et al. The aryl hydrocarbon receptor nuclear translocator is an essential regulator of murine hematopoietic stem cell viability. Blood. 2015;125(21):3263-3272.

57. Koong AC, Chen EY, Mivechi NF, Denko NC, Stambrook P, Giaccia AJ. Hypoxic activation of nuclear factor-kappa $\mathrm{B}$ is mediated by a Ras and Raf signaling pathway and does not involve MAP kinase (ERK1 or ERK2). Cancer Res. 1994;54(20):5273-5279.

58. Oliver KM, et al. Hypoxia activates NF-kappaB-dependent gene expression through the canonical signaling pathway. Antioxid Redox Signal. 2009;11(9):2057-2064.

59. Hayden MS, Ghosh S. NF- $\mathrm{B}$, the first quarter-century: remarkable progress and outstanding questions. Genes Dev. 2012;26(3):203-234.

60. Cummins EP, et al. Prolyl hydroxylase-1 negatively regulates IkappaB kinase-beta, giving insight into hypoxia-induced NFkappaB activity. Proc Natl Acad Sci USA. 2006;103(48):18154-18159.

61. Fu J, Taubman MB. Prolyl hydroxylase EGLN3 regulates skeletal myoblast differentiation through an NF-kappaB-dependent pathway. J Biol Chem. 2010;285(12):8927-8935.

62. Zheng X, et al. Prolyl hydroxylation by EglN2 destabilizes FOXO3a by blocking its interaction with the USP9x deubiquitinase. Genes Dev. 2014;28(13):1429-1444.

63. Cockman ME, et al. Posttranslational hydroxylation of ankyrin repeats in IkappaB proteins by the hypoxia-inducible factor (HIF) asparaginyl hydroxylase, factor inhibiting HIF (FIH). Proc Natl Acad Sci USA . 2006;103(40):14767-14772.

64. Scholz CC, et al. FIH regulates cellular metabolism through hydroxylation of the deubiquitinase OTUB1. PLoS Biol. 2016;14(1):e1002347.

65. Hams E, et al. The hydroxylase inhibitor dimethyloxallyl glycine attenuates endotoxic shock via alternative activation of macrophages and IL-10 production by B1 cells. Shock. 2011;36(3):295-302.

66. Scholz CC, et al. Regulation of IL-1 $\beta$-induced $\mathrm{NF}-\kappa \mathrm{B}$ by hydroxylases links key hypoxic and inflammatory signaling pathways. Proc Natl Acad Sci USA. 2013;110(46):18490-18495.

67. D'Ignazio L, Bandarra D, Rocha S. NF- $\kappa B$ and HIF crosstalk in immune responses. FEBS J. 2016;283(3):413-424.

68. Bruning U, Fitzpatrick SF, Frank T, Birtwistle M, Taylor CT, Cheong A. NFкB and HIF display synergistic behaviour during hypoxic inflammation. Cell Mol Life Sci. 2012;69(8):1319-1329.

69. Görlach A, Bonello S. The cross-talk between NF-kappaB and HIF-1: further evidence for a significant liaison. Biochem J. 2008;412(3):e17-e19.

70. Taylor CT. Interdependent roles for hypoxia inducible factor and nuclear factor-kappaB in hypoxic inflammation. J Physiol (Lond). 2008;586(17):4055-4059.

71. Bonello S, et al. Reactive oxygen species activate the HIF-1alpha promoter via a functional NFkappaB site. Arterioscler Thromb Vasc Biol. 2007;27(4):755-761.

72. van Uden P, Kenneth NS, Rocha S. Regulation of hypoxia-inducible factor-1alpha by NF-kappaB. Biochem J. 2008;412(3):477-484.

73. Frede S, Stockmann C, Freitag P, Fandrey J. Bacterial lipopolysaccharide induces HIF-1 activation in human monocytes via p44/42 MAPK and NF-kappaB. Biochem J. 2006;396(3):517-527.

74. Rius J, et al. NF-kappaB links innate immunity to the hypoxic response through transcriptional regulation of HIF-1alpha. Nature. 2008;453(7196):807-811.

75. Jaakkola P, et al. Targeting of HIF-alpha to the von Hippel-Lindau ubiquitylation complex by $\mathrm{O}_{2}$-regulated prolyl hydroxylation. Science. 2001;292(5516):468-472.

76. Ivan M, et al. HIFalpha targeted for VHL-mediated destruction by proline hydroxylation: implications for $\mathrm{O}_{2}$ sensing. Science. 2001;292(5516):464-468.

77. Mahon PC, Hirota K, Semenza GL. FIH-1: a nove protein that interacts with HIF-1alpha and VHL to mediate repression of HIF-1 transcriptional activity. Genes Dev. 2001;15(20):2675-2686.

78. Lando D, Peet DJ, Whelan DA, Gorman JJ, Whitelaw ML. Asparagine hydroxylation of the HIF transactivation domain a hypoxic switch. Science. 2002;295(5556):858-861.

79. Rodriguez J, et al. Substrate-trapped interactors of PHD3 and FIH cluster in distinct signaling pathways. Cell Rep. 2016;14(11):2745-2760.

80. Takeda Y, et al. Macrophage skewing by Phd 2 haplodeficiency prevents ischaemia by inducing arteriogenesis. Nature. 2011;479(7371):122-126.

81. Ikeda J, et al. Deletion of phd 2 in myeloid lineage attenuates hypertensive cardiovascular remodeling. J Am Heart Assoc. 2013;2(3):e000178.

82. Swain L, et al. Prolyl-4-hydroxylase domain 3
(PHD3) is a critical terminator for cell survival of macrophages under stress conditions. J Leukoc Biol. 2014;96(3):365-375.

83. Kiss J, et al. Loss of the oxygen sensor PHD3 enhances the innate immune response to abdominal sepsis. J Immunol. 2012;189(4):1955-1965.

84. Walmsley SR, et al. Prolyl hydroxylase 3 (PHD3) is essential for hypoxic regulation of neutrophilic inflammation in humans and mice. JClin Invest. 2011;121(3):1053-1063.

85. Tambuwala MM, et al. Loss of prolyl hydroxylase-1 protects against colitis through reduced epithelial cell apoptosis and increased barrier function. Gastroenterology. 2010;139(6):2093-2101.

86. Chen Y, et al. PHD3 stabilizes the tight junction protein occludin and protects intestinal epithelial barrier function. J Biol Chem. 2015;290(33):20580-20589.

87. Van Welden S, Laukens D, Ferdinande L, De Vos M, Hindryckx P. Differential expression of proly hydroxylase 1 in patients with ulcerative colitis versus patients with Crohn's disease/infectious colitis and healthy controls. J Inflamm (Lond). 2013;10(1):36.

88. Kalucka J, et al. Loss of epithelial hypoxia-inducible factor prolyl hydroxylase 2 accelerates skin wound healing in mice. Mol Cell Biol. 2013;33(17):3426-3438.

89. Mamlouk S, et al. Loss of prolyl hydroxylase-2 in myeloid cells and T-lymphocytes impairs tumor development. Int J Cancer. 2014;134(4):849-858.

90. O'Neill LA, Hardie DG. Metabolism of inflammation limited by AMPK and pseudo-starvation. Nature. 2013;493(7432):346-355.

91. Elsby LM, Donn R, Alourfi Z, Green LM, Beaulieu E, Ray DW. Hypoxia and glucocorticoid signaling converge to regulate macrophage migration inhibitory factor gene expression. Arthritis Rheum. 2009;60(8):2220-2231.

92. Leonard MO, Godson C, Brady HR, Taylor CT. Potentiation of glucocorticoid activity in hypoxia through induction of the glucocorticoid receptor. Jimmunol. 2005;174(4):2250-2257.

93. Ameri K, Harris AL. Activating transcription factor 4. Int J Biochem Cell Biol. 2008;40(1):14-21.

94. Taylor CT. Regulation of intestinal epithelial gene expression in hypoxia. Kidney Int. 2004;66(2):528-531.

95. Reiling JH, Sabatini DM. Stress and mTORture signaling. Oncogene. 2006;25(48):6373-6383.

96. Mekhail K, Gunaratnam L, Bonicalzi ME, Lee S. HIF activation by $\mathrm{pH}$-dependent nucleolar sequestration of VHL. Nat Cell Biol. 2004;6(7):642-647.

97. Lee DC, et al. A lactate-induced response to hypoxia. Cell. 2015;161(3):595-609.

98. Fábián Z, Taylor CT, Nguyen LK. Understanding complexity in the HIF signaling pathway using systems biology and mathematical modeling. JMol Med. 2016;94(4):377-390.

99. Ryan S, Taylor CT, McNicholas WT. Selective activation of inflammatory pathways by intermittent hypoxia in obstructive sleep apnea syndrome. Circulation. 2005;112(17):2660-2667.

100. Taylor CT, Kent BD, Crinion SJ, McNicholas WT, Ryan S. Human adipocytes are highly sensitive to intermittent hypoxia induced NF-kappaB activity and subsequent inflammatory gene expression. Bio- 
chem Biophys Res Commun. 2014;447(4):660-665.

101.Prabhakar NR, Semenza GL. Adaptive and maladaptive cardiorespiratory responses to continuous and intermittent hypoxia mediated by hypoxia-inducible factors 1 and 2. Physiol Rev. 2012;92(3):967-1003.

102. Nanduri J, et al. HIF-1 $\alpha$ activation by intermittent hypoxia requires NADPH oxidase stimulation by xanthine oxidase. PLOS ONE. 2015;10(3):e0119762.

103. Cummins EP, Doherty GA, Taylor CT. Hydroxylases as therapeutic targets in inflammatory bowel disease. Lab Invest. 2013;93(4):378-383.

104.Cummins EP, et al. The hydroxylase inhibitor dimethyloxalylglycine is protective in a murine model of colitis. Gastroenterology. 2008;134(1):156-165.

105. Robinson A, Keely S, Karhausen J, Gerich ME, Furuta GT, Colgan SP. Mucosal protection by hypoxia-inducible factor prolyl hydroxylase inhibition. Gastroenterology. 2008;134(1):145-155.

106. Taylor CT, Colgan SP. Hypoxia and gastrointestinal disease. J Mol Med. 2007;85(12):1295-1300.

107. Jamadarkhana $P$, et al. Treatment with a novel hypoxia-inducible factor hydroxylase inhibitor (TRC160334) ameliorates ischemic acute kidney injury. Am J Nephrol. 2012;36(3):208-218.

108.Schaffer K, Taylor CT. The impact of hypoxia on bacterial infection. FEBS J. 2015;282(12):2260-2266.
109. Schaible B, et al. Hypoxia modulates infection of epithelial cells by Pseudomonas aeruginosa. PLOS ONE. 2013;8(2):e56491.

110. Berger EA, McClellan SA, Vistisen KS, Hazlett LD. HIF- $1 \alpha$ is essential for effective PMN bacterial killing, antimicrobial peptide production and apoptosis in Pseudomonas aeruginosa keratitis. PLoS Pathog. 2013;9(7):e1003457.

111. Peyssonnaux C, Boutin AT, Zinkernagel AS, Datta V, Nizet V, Johnson RS. Critical role of HIF-1alpha in keratinocyte defense against bacterial infection. J Invest Dermatol. 2008;128(8):1964-1968.

112. Zinkernagel AS, Peyssonnaux C, Johnson RS, Nizet V. Pharmacologic augmentation of hypoxia-inducible factor-1alpha with mimosine boosts the bactericidal capacity of phagocytes. JInfect Dis. 2008;197(2):214-217.

113. Okumura CY, et al. A new pharmacological agent (AKB-4924) stabilizes hypoxia inducible factor-1 (HIF-1) and increases skin innate defenses against bacterial infection. J Mol Med. 2012;90(9):1079-1089.

114. Hirota SA, et al. Hypoxia-inducible factor signaling provides protection in Clostridium difficile-induced intestinal injury. Gastroenterology. 2010;139(1):259-69.e3.

115. Elks PM, et al. Hypoxia inducible factor signaling modulates susceptibility to mycobacterial infection via a nitric oxide dependent mechanism. PLoS Pathog. 2013;9(12):e1003789.
116. Chan MC, Holt-Martyn JP, Schofield CJ, Ratcliffe PJ. Pharmacological targeting of the HIF hydroxylases - a new field in medicine development. MolAspects Med. 2016;47-48:54-75.

117. Besarab A, et al. Roxadustat (FG-4592): Correction of anemia in incident dialysis patients. J Am Soc Nephrol. 2016;27(4):1225-1233.

118. Holdstock L, et al. Four-week studies of oral hypoxia-inducible factor-prolyl hydroxylase inhibitor GSK1278863 for treatment of anemia. JAm Soc Nephrol. 2016;27(4):1234-1244.

119. Tambuwala MM, Manresa MC, Cummins EP, Aversa V, Coulter IS, Taylor CT. Targeted delivery of the hydroxylase inhibitor DMOG provides enhanced efficacy with reduced systemic exposure in a murine model of colitis. JControl Release. 2015;217:221-227.

120. Kamm MA, et al. Once-daily, high-concentration MMX mesalamine in active ulcerative colitis. Gastroenterology. 2007;132(1):66-75; quiz 432.

121. Travis SP, et al. Once-daily budesonide MMX in active, mild-to-moderate ulcerative colitis: results from the randomised CORE II study. Gut. 2014;63(3):433-441.

122. Cummins EP, et al. NF- $\mathrm{B}$ links $\mathrm{CO}_{2}$ sensing to innate immunity and inflammation in mammalian cells. JImmunol. 2010;185(7):4439-4445.

123. Taylor CT, Cummins EP. Regulation of gene expression by carbon dioxide. J Physiol (Lond). 2011;589(Pt 4):797-803. 